Entwicklungspotentiale des Umweltmanagements bei ökologischen Pionierunternehmen

\section{Die Macht der weichen Faktoren}

\section{In der zweiten und höheren Phase ökologischer Unternehmenspolitik sind eher weiche Faktoren der Unternehmensführung ausschlaggebend für weitere Erfol- ge zur Entlastung der Umwelt. Um sie zu erfassen und zielgerichtet weiter- entwickeln zu können, wurde die Methodik des Soft Factor Assessments neu entwickelt.}

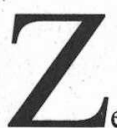
entrale Ausgangsthese des Projektes (1) war, daß sich betrieblicher Umweltschutz in einer ersten Phase auf technische Faktoren stützt, mit dem Ziel einer kurzfristigen Umweltentlastung. In der Literatur der letzten Jahre zum Thema Umweltmanagement wurden hierfür verschiedenste Instrumente dargestellt. Eine langfristige Perspektive umweltschutzbezogener Entwicklung von Unternehmen läßt sich unserer Meinung nach aber nur über die Integration weicher Faktoren in den Prozeß der Umweltentlastung erreichen.

In einem ersten Schritt des Projektes kristallisierten sich sowohl durch eine Literaturrecherche als auch über einen Pretest bei den beteiligten Unternehmen (1) eine Reihe von weichen Faktoren heraus, die entsprechend dem Forschungsstand relefähigkeit von Unternehmen sind: vant für die ökologische Entwicklungs-
Visionsfähigkeit: Unternehmensbezogene Visionen können sowohl allgemein als auch ökologisch bezogen einen wichtigen Beitrag zur Sinnstiftung leisten. Grundgedanke ist daher die Annahme, daß eine umweltschutzbezogene zielgerichtete Entwicklung langfristig nur durch die Formulierung visionärer Entwicklungsziele möglich ist.

- Fähigkeit zur Zielentwicklung: Damit ökologische Visionen nicht unbeachtet neben dem betrieblichen Alltag stehen, müssen sie zu konkreten Zielvorstellungen umformuliert werden, um eine zielgerichtete Entwicklung zu ermöglichen.

- Fähigkeit zur Organisation von Umweltschutz: Dieser Faktor bezieht sich nicht auf das Vorhandensein einer betrieblichen Umweltschutzorganisation. Vielmehr ist hier die Fähigkeit angesprochen, neue, sich ständig wandelnde Herausforderungen und Probleme in alltägliche Handlungsroutinen aufzunehmen.

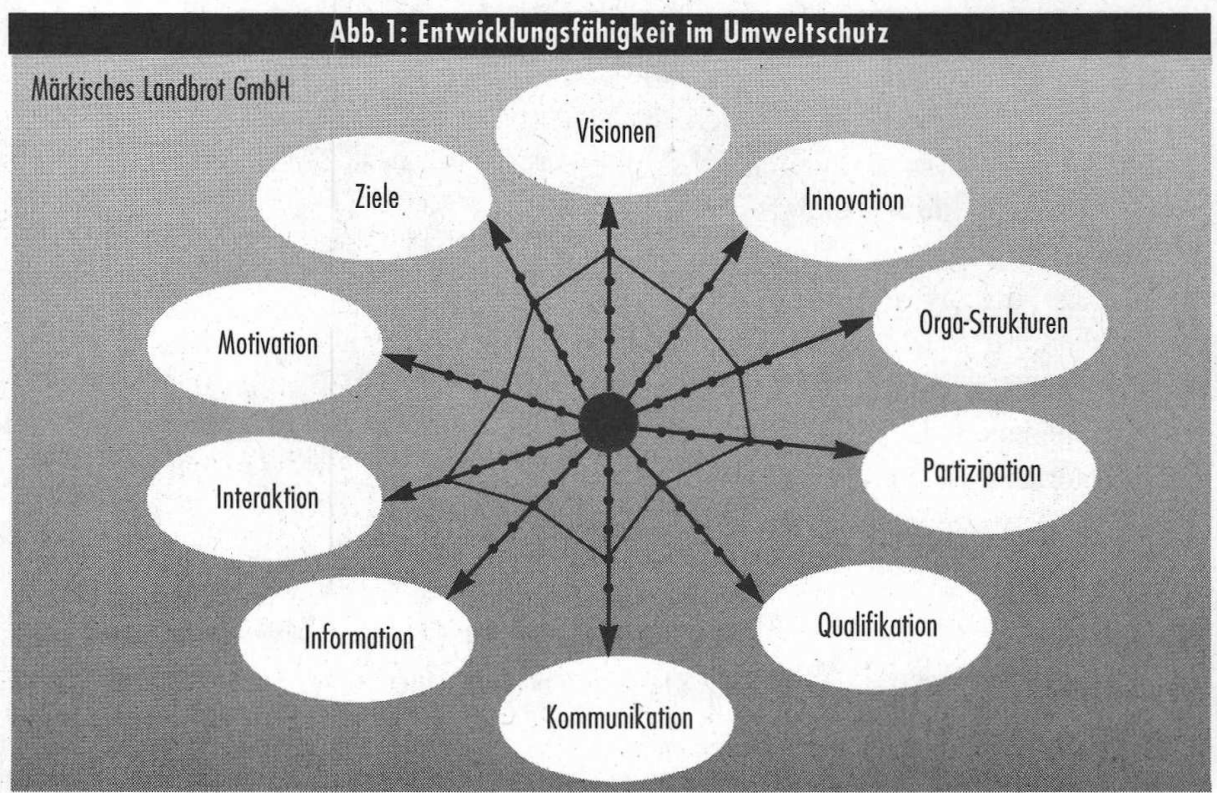

Um dem Wesen ökologischer Probleme gerecht $\mathrm{zu}$ werden, ist dazu ein großes $\mathrm{Maß}$ an Flexibilität notwendig.

- Innovationsfähigkeit der Gesamtorganisation des Unternehmens: Eine Weiterentwicklung der Gesamtorganisation des Unternehmens bedingt natürlich die Integration der Mitarbeiter, da sie wesentlich die Kapazitäten des Unternehmens darstellen. Dieser Faktor beleuchtet die Fragestellung, inwiefern ein Unternehmen in der Lage ist, Rahmenbedingungen für eine Integration der Mitarbeiter in den kontinuierlichen ökologischen Entwicklungsprozeß zu schaffen.

- Fähigkeit zur Kommunikation wesentlicher Aspekte des Umweltschutzes: Dieser Faktor bezieht sich auf die Fähigkeit eines Unternehmens relevante ökologische Informationen $\mathrm{zu}$ beschaffen und wahrzunehmen sowie die Möglichkeiten entsprechend $\mathrm{zu}$ kommunizieren.

- Fähigkeit zur Interaktion: Wesentlich für eine umweltschutzbezogene Entwicklung sind die Impulse, die von außen an das Unternehmen herangetragen werden. Dieser Faktor zielt auf die Art und Weise, wie das Unternehmen ökologische Anforderungen, die von außen gestellt werden, wahrnimmt und welche Konsequenzen dies für das Verhalten des Unternehmens nach außen hat.

- Fähigkeit zur Förderung des Mitarbeiterpotentials: Dieser Faktor zielt nicht so sehr auf eine Qualifikation im Sinne von Wissensvermittlung ab. Vielmehr meint dieser Faktor eine weitreichende Personalentwicklung, die den besonderen Anforderungen der ökologischen Herausforderung Rechnung trägt.

- Normen und Wertebilder: Auch in Unternehmen läßt sich oft eine Differenz zwischen ökologischen Einstellungen und Verhalten feststellen. Dieser Faktor beinhaltet die Frage, inwieweit soziale Normen innerhalb des Unternehmens ökologisches Verhalten beeinflussen.

\section{Soft Factor Assessment}

Auf Basis der oben dargestellten Faktoren wurde eine Methodik erarbeitet, die es den Unternehmen ermöglicht, ihre Ausprägung der weichen Faktoren zu 


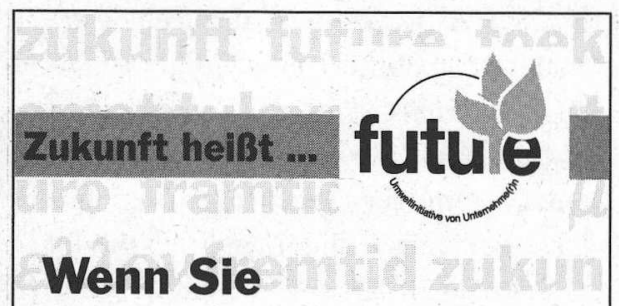

\section{verantwortungsbewuBt wirtschaften wollen ...}

\section{... auf die Entwicklung Innovativer und kreativer Konzepte setzen ...}

\section{iningt... ökologisch und wirtschaftlich handeln müssen ...}

\section{... eine umweltorientierte und sozial verpfilchtete Marktwirtschaft gestalten möchten ...}

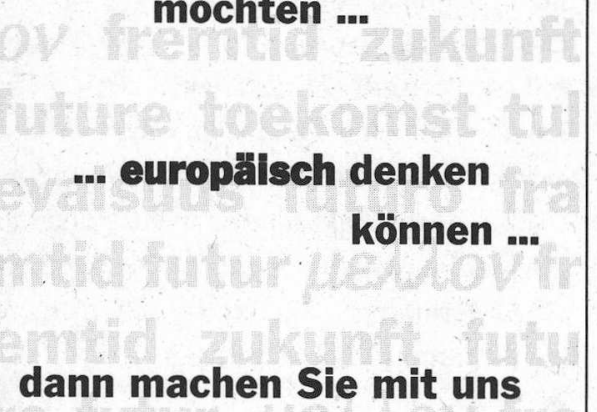

\section{die Zukunft zur Chance!}

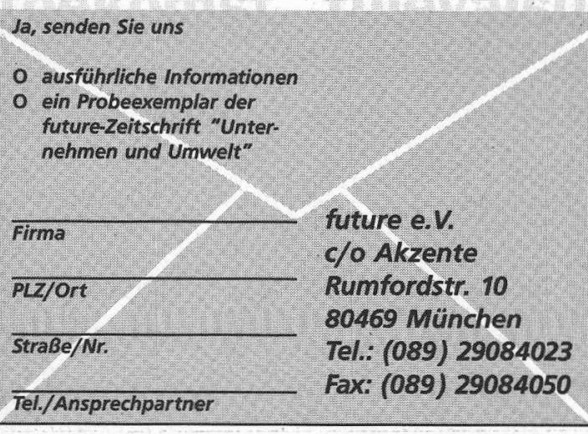

erfassen. Darüber hinaus bietet die Methodik Ansatzpunkte zu einer weiteren Entwicklung der weichen Faktoren. Ziel des Soft Factor Assessments ist es nicht, konkrete Maßnahmen vorzugeben, sondern Hilfestellungen auf dem Weg zu einer ökologischen Entwicklungsfähigkeit zu geben. Es ist daher so konzipiert, daß es von jedem Unternehmen - unabhängig vom umweltschutzbezogenen Entwicklungsstand - durchgeführt werden kann. Ebenso bietet das Soft Factor Assessment die Möglichkeit, bisherige Maßnahmen zu evaluieren. Hierdurch erhält das Unternehmen einen Überblick über die Wirkung der bisher umgesetzten Maßnahmen.

Unternehmen sollen durch die Anwendung des Soft Factor Assessments in die Lage versetzt werden, einen ständigen Reflexions- und Lernprozeß im Umweltschutz zu initiieren.

In der ersten Phase werden die Einstellungen der Mitarbeiter und der Unternehmensleitung zu den weichen Faktoren erhoben. Dies erfolgt durch die Anwendung von Polaritätsprofilen. Die Auswertung wird dann - wie die Abbildung auf Seite 19 zeigt - entsprechend graphisch aufbereitet. Diese Ist-Analyse zeigt dem Unternehmen den bisher erreichten Stand.

In einem nächsten Schritt werden Einflußfaktoren erarbeitet, die zukünftig extern auf das Unternehmen einwirken. Diese Faktoren werden im folgenden dann hinsichtlich ihrer Wirkung auf das Unternehmen bewertet. Der Betrieb kann somit auf eine interne Ist-Analyse wie auch auf eine Analyse hinsichtlich der externen Faktoren zurückgreifen.

Erst diese beiden Analysebereiche zusammen versetzen die Unternehmung in die Lage, die zukünftigen Anforderungen, die an das Unternehmen gestellt werden, mit den vorhandenen Fähigkeiten abzugleichen. Hierdurch bietet sich dem Unternehmen ein Ansatzpunkt hinsichtlich der weiteren ökologischen Entwicklungspotentiale.

\section{Fazit}

Die an dem Projekt beteiligten Unternehmen gehören zu den Vorreiterfirmen hin- sichtlich des Umweltschutzes. Ihre Aktivitäten beziehen sich nicht nur auf technische und ökonomische Bereiche sondern auch auf die hier erläuterten weichen Faktoren. Es kann aber insgesamt festgestellt werden, daß eine systematische Betrachtung und Analyse der weichen Faktoren nur in Ansätzen vorliegt. Dementsprechend wurde die in diesem Projekt erarbeitete Methodik nicht nur sehr begrüßt, die Unternehmen haben sich auch mit großem Interesse an der Ausarbeitung beteiligt. Erste Ansätze, die Methodik in das eigene Unternehmen zu integrieren, sind bereits vorhanden.

Insgesamt läßt sich festhalten, daß eine Integration der weichen Faktoren in die ökologische Unternehmenspolitik vielfach noch nicht Realität geworden ist. Um aber Unternehmen, die sich bisher kaum mit betrieblichem Umweltschutz beschäftigt haben, einen Ansatzpunkt zur Erfassung und zielgerichteten Entwicklung von weichen Faktoren im Umweltschutz zu geben, soll in einem weiteren Projekt die Methodik so weiterentwickelt werden, daß sie von jedem Unternehmen angewandt werden kann.

\section{Anmerkungen}

(1) Das Projekt zur ökologischen Entwicklungsfähigkeit im Auftrag der Deutschen Bundesstiftung Umwelt wurde in enger Kooperation mit elf Unternehmen durchgeführt, die als ökologische Vorreiterfirmen gelten.

Folgende Unternehmen waren am Projekt beteiligt: Bosch-Siemens Hausgeräte GmbH - Standort Dillingen; Gebhardt Ventilatoren GmbH \& C 0 ., Waldenburg; Georg Kohl GmbH \& Co., Brackenheim; Ludwig Stocker Hofpfisterei GmbH, München; Kunert AG - Standort Immenstadt; Märkisches Landbrot GmbH, Berlin; Merckle/ratiopharm GmbH Standort Ulm; Oktoberdruck GmbH, Berlin; Siemens-Nixdorf Informationssysteme AG - Standort Paderborn; Sodasan GmbH, Apen-Nordloh; Wilkhahn-Wilkening und Hahne GmbH \& Co., Bad Münder

\section{Die Autorlnnen}

Carsten Gellrich ist geschüftstührender Gesellschafter der ecco ecology \& communication Unternehmensberatung $\mathrm{GmbH}$, Alexandra Luig Mitarbeiterin am Projekt zur ökologischen Entwicklungsfähigkeit. Kontakt: ecco Unternehmensberatungs $\mathrm{GmbH}$, Augusistr. 88, 26121 Oldenburg, (0441) 779059, e-mail luig@ecco.de 
(c) 20I0 Authors; licensee IÖW and oekom verlag. This is an article distributed under the terms of the Creative Commons Attribution Non-Commercial No Derivates License (http://creativecommons.org/licenses/by-nc-nd/3.o/), which permits unrestricted use, distribution, and reproduction in any medium, provided the original work is properly cited. 